\title{
Las Tecnologías de la Información y Comunicación y la Brecha Digital: Una nueva forma de exclusión social
}

\author{
Information and Communication Technologies and the Digital Divide: A new form of social \\ exclusion
}

\author{
José F. Hernández - Gracia ${ }^{a}$, Venancio Avendaño - Hernández ${ }^{b}$ Hugo A. Buitrón-Ramírez ${ }^{c}$
}

\begin{abstract}
:
Lorem Let's start by understanding that technology is any knowledge, skill or competence that can be used systematically to achieve a specific objective. On the other hand, technological innovation refers to the incorporation of new technologies to improve changes in production, in business and in new forms of work. Then, companies are related in a technological and global world, whose modality is digitalization, networking and hyper connection that allows people located anywhere in the world to be a customer or user of goods and services immediately. Digitization offers companies development and efficiency, this is a reality that causes the rhythms of each organization at the time of adopting it to be very different, being able to produce dysfunctions within the company during the process. In other words, while technology changes very rapidly, organizations can not always follow the same pace, and that is precisely when the digital divide in the company itself occurs.
\end{abstract}

Keywords:

Technology, digitalization, development, digital divide

\section{Resumen:}

Empecemos entendiendo que la tecnología es cualquier conocimiento, habilidad o competencia que se puede emplear de manera sistemática para lograr un objetivo determinado. Por otro lado, la innovación tecnológica se refiere a la incorporación de nuevas tecnologías que permitan mejorar cambios en la producción, en los negocios y en las nuevas formas de trabajo. Luego entonces las empresas se relacionan en un mundo tecnológico y global, cuya modalidad es la digitalización, el trabajo en red y la híper conexión que permite que las personas ubicadas en cualquier parte del mundo pueda ser cliente o usuario de bienes y servicios de forma inmediata. La digitalización ofrece a las empresas desarrollo y eficiencia, esta es una realidad que provoca que los ritmos de cada organización a la hora de adoptarla sean muy diferentes, pudiéndose producir disfunciones dentro de la propia empresa durante el proceso. Es decir, que mientras la tecnología cambia muy rápidamente, las organizaciones no siempre pueden seguir el mismo ritmo, y es ahí precisamente cuando se produce la brecha digital en la propia empresa.

\section{Palabras Clave:}

Tecnología, digitalización, desarrollo, brecha digital

\section{Introducción}

Es importante destacar que digitalizar la información, se refiere al proceso de transformar toda la información analógica a un formato digital para que pueda ser almacenada, consultada y manipulada durante las actividades y procesos de las empresas. No obstante, hoy en día existe una gran brecha digital, no solo en la empresa, también en las personas que no logran adaptarse a estos cambios tecnológicos, quedando en desventaja contra aquellos que, si logran adaptarse. En este trabajo encontraremos información acerca de esta brecha digital, de los factores que impiden una verdadera inclusión, así como el fenómeno que se refiere a la adicción que esta causando revuelo en la tecnología.

\footnotetext{
a Autor de Correspondencia. Universidad Tecnológica de Tula - Tepeji, ORCID: 0000-0001-5712-3018, Email: josefrancisco.hernandez@uttt.edu.mx

${ }^{\text {b }}$ Universidad Tecnológica de Tula - Tepeji, ORCID: 0000-0001-5577-5610, Email: venancio.avendano@uttt.edu.mx

• Universidad Tecnológica de Tula - Tepeji, Email: hugoarmando.buitron@uttt.edu.mx
} 


\section{La presencia de la brecha digital en las empresas.}

Según (1) establecen que las herramientas tecnológicas se han convertido en un factor indispensable para alcanzar mejores condiciones de bienestar y desarrollo entre la Sociedad, Empresa y Gobierno. Situación que no podemos negar, independientemente del país, región o localidad en la que nos encontremos, sin embargo, se ha observado y estudiado las divisiones que ha generado entre los que han alcanzado un nivel avanzado de desarrollo y los que no, entre los que construyen su poder económico en las nuevas tecnologías y entre los que se ven rezagados ante la falta de acceso a la información, educación o investigación científica (2). Bajo este tenor, se entiende que dicha división representa la llamada "brecha digital", que no es más que otra consecuencia de las desigualdades sociales del mundo, y que se caracteriza por la inequidad antepuesta a los individuos en las esferas del acceso, uso y apropiación de las TIC.

Pero, ¿qué genera la brecha digital? Se sabe que es una expresión de desigualdad, y que (2) explica atinadamente, por las diferencias generacionales, falta de capacitación en el uso de las tecnologías, el mismo rechazo cultural a estas herramientas y la carencia de beneficios específicos. A este listado, nosotros le agregamos también, la resistencia al cambio, y de forma paralela, a la falta de transmisión del conocimiento (Brecha Cognitiva). Esta última tiene que ver con las habilidades de las personas para el manejo de las TIC, y la imposibilidad de transformar la gran cantidad de información disponible hoy día en conocimiento.

Ahora bien, el problema que nos atañe es la presencia de la mencionada brecha digital en las empresas y en nuestros puestos de trabajo, la cual se hace notable ante la falta de capacidad de respuesta de algunas organizaciones para subirse a la ola del desarrollo tecnológico; como pueden ser la inversión en herramientas productivas como el Cloud Computing, Big Data, Realidad Virtual, Realidad Aumentada, Inteligencia Artificial o Impresión 3D, que requieren de gran financiamiento y de conocimiento técnico especial. Por lo tanto, se abre una división donde la alta especialización y la implementación de dichas tecnologías exigen nuevas competencias y habilidades que actualmente están afectando a las PyMes en México. Demuner y Becerril y Nava, en su trabajo identificaron el entorno en que se desarrollaban las PyMes en relación a la adopción de TIC en México, los resultados resaltan la amplia brecha digital de estas mismas en la adopción de las TIC; y dado a que su implementación se ha dado aún de manera incipiente, donde las empresas más grandes hacen mayor uso de éstas y las pequeñas no aprovechan los beneficios de dichas tecnologías (3).
Por otro lado, no todo se debe a factores exógenos hacia las empresas más rezagadas, sino que también se ha evidenciado que algunas PyMes se han resistido al cambio; ejemplo de ello es el observado cuando se puso en marcha la reforma fiscal en el 2014, muchas de ellas se mostraron reticentes ante la nueva normativa de la facturación electrónica por la falta de la tecnología necesaria, considerándola un gasto innecesario y no una inversión estratégica para sus operaciones (4).

Asimismo, es claro y evidente que las nuevas tecnologías están reemplazando puestos de trabajos tradicionales, que si bien propician la generación de otros nuevos, no se están creando en la misma medida que se desea (5). Lo cual implica que las personas que son despedidas de su trabajo y en su casa no cuentan con conexión a Internet o con PC, se quedan sin dicho empleo y a la vez se quedan más rezagadas con respecto al uso de las tecnologías del ambiente laboral. Pero del otro lado, cuando se trata de utilizar determinada tecnología de vanguardia no todos los colaboradores implicados en su uso tienen los conocimientos o la voluntad para acceder a dicho cambio, lo cual termina por multiplicar y repetir funciones en las que se desaprovechan tiempo, recursos materiales y humanos, y se invalida por completo el intento de modernización propuesto (6). Situación similar, es la observada con las diferencias generacionales, tanto jóvenes como adultos, pueden ser proclives o no, de hacer un buen uso de las TIC en el ambiente de trabajo o educativo. Es verdad que los jóvenes son más asiduos a las redes sociales como Facebook, Twitter, Instagram, etc.; pero contrario a lo que muchos piensan, si trasladamos el uso de dichas herramientas al trabajo, no saben cómo comportarse o sacarles un mayor provecho fuera de lo que están acostumbrados, ya que consideran que sólo sirven para comunicarse con sus amigos, no con un cliente $o$ con sus jefes (7).

Con base a lo anterior, opinamos que para reducir la brecha digital en la empresa, debe ser un trabajo conjunto entre los líderes y sus colaboradores, así como con el gobierno para fomentar la apropiación de las nuevas tecnologías, propiciar su uso en la vida cotidiana y laboral de forma responsable, así como establecer las fuentes de financiamiento correspondientes.

\section{Impedimentos se dan para lograr la verdadera inclusión digital.}

La brecha digital no solo se da en las empresas, sino también de manera muy importante en las personas que no logran adaptarse a estos cambios tecnológicos, quedando en desventaja contra aquellos que, si logran adaptarse, por lo que entonces se debe considerar que la brecha digital es una nueva expresión de desigualdad, que se da por los siguientes factores (8): 
- Costo de acceso, que se refiere al bajo poder adquisitivo de la población.

- Telecomunicaciones, el desarrollo de políticas públicas que permitan un mejor acceso global a la tecnología.

- La relación entre cultura y tecnología, cambio de paradigmas, de costumbres, de ideología, etc.

- Así como la manera de obtener el conocimiento y desarrollo de habilidades tecnológicas.

- Otro de los impedimentos importantes es la brecha digital de género, donde a pesar de que las nuevas generaciones de mujeres presentan actitudes diferentes los avances aún no se equilibran con respecto al dominio epistemológico de los hombres.

- Tal como ocurre con otros problemas sociales complejos, en primer lugar la verdadera inclusión digital es un problema de carácter multifactorial, por lo que no es atribuible a un fenómeno único.

- La mejor forma en que se puede comprender este problema multifactorial, es agrupar en diferentes factores las causas que fomentan la exclusión digital:

$\checkmark$ Factores sociales. En primera instancia, observamos que la exclusión digital tiene una fuerte relación con la desigualdad social, se sabe sin lugar a dudas que una familia que vive al día a día y que difícilmente consigue los recursos para alimentarse, ni siquiera tendrá como prioridad o interés acceder a servicios digitales o conexión a red.

$\checkmark$ Factores económicos. En esta vertiente percibimos que además de la desigualdad social explicada previamente, existen fuertes intereses económicos por fortalecer infraestructura digital en áreas estratégicas que generarán grandes volúmenes de negocio para compañías importantes, lo que conlleva nuevamente a un olvido entre las comunidades que no representen negocio.

$\checkmark \quad$ Factores políticos. En este apartado nos remitimos a la historia del País donde cíclicamente se ha notado que un pueblo desinformado es más fácil de controlar, por ello se presume que contar con una conectividad democrática o igualitaria podría llevar a diferentes resultados en momentos políticos. $\checkmark$ Factores tecnológicos. Esta área que tiene además de una necesaria vinculación con los factores anteriores, se relaciona más intensamente con el ramo económico, nuestro país debido a su extensión, gran cantidad de climas y ubicación de poblaciones en zonas muy alejadas, sería extremadamente caro enlazar todos los rincones del país con tecnología de punta de lanza.

$\checkmark$ Factores educativos. Finalmente, y no menos importante observamos que si todos los factores anteriores se resolvieran, todavía se tendría que eliminar el alfabetismo tecnológico en el país, sea por medio de facilitadores 0 tutores o profesores, etc. De tal suerte que el uso de los servicios digitales, la conectividad, etc. tuvieran un significado y utilidad en la vida de todos aquellos que están excluidos actualmente.

Factores demográficos. Conforme la población menos favorecida siga creciendo la brecha digital sólo se irá acentuando, porque no se ha abonado en pro de la solución.

\section{Las adicciones a la tecnología en el ámbito empresarial.}

El termino "adicción al internet" no esta considerado como tal por la Asociación Americana de Psiquiatría y tampoco ha sido aceptada por la Asociación Americana de Psicología. Hasta hoy no hay nada que oficialice esta adicción, no obstante, a las personas se les ha despedido de su trabajo por el uso excesivo de esta actividad, entonces habrán de buscar las causas reales de éste comportamiento. El uso intensivo del internet se pudiera deber a cambios de forma de ser o pensar, de nuevas tendencias 0 en busca de un mayor conocimiento. Algunas manifestaciones adictivas podrían ser: La obsesión por perderse algo de lo que está sucediendo en la red, la adicción al whatsapp, ansiedad al teléfono celular, la utilización enfermiza del smasrtphone, entre otros. En más problemas negativos a considerar serían: El estrés tecnológico, sobrecarga de información, multitarea, adicciones y uso indebido de la tecnología. En fin que La investigación necesita definir claramente la dependencia de la tecnología, la adicción y el uso excesivo para que haya una gran comprensión de lo que se quiere decir. Los recursos tecnológicos son imparciales y de acuerdo al empleo que les demos podrían generar efectos a favor o en contra de los entes sociales o empresariales, debiendo entender que las TIC son un medio que hace más fácil el trabajo y la vida más agradable, por lo que es conveniente obtener el mejor 
provecho de éstas, impidiendo que su uso cause desperfectos. Lo maravilloso sería que las pusiéramos a nuestro servicio y no al contrario. Una vez que el trabajo se lleva a la vida privada, las personas se vuelven irritables y tienden a ser más propensas a sufrir enfermedades y/o accidentes causados por el deterioro en su calidad de vida (9). Los continuos cambios tecnológicos y el permanente proceso de aprendizaje de los descubrimientos al que se someten las personas, favorecen la aparición del tecnoestrés. Por otra parte, definir la adicción tecnológica nos conduce a varios enfoques en los que no se tiene un consenso general, debido a que se puede ver como uso excesivo o malos hábitos en el manejo de los medios digitales. Por lo que deducir cómo impactarán las connotaciones antes mencionadas al medio empresarial resulta confuso y peligroso. En las últimas décadas la tecnología ha provocado un gran cambio en la sociedad, produce grandes beneficios en la vida de las personas, pero también hay datos que deben alertar los riesgos derivados de tantas horas utilizando las nuevas tecnologías en la vida cotidiana de las personas. Según la Asociación de Internet. Mx. (2018), los usuarios pasan 13 horas al día frente a los medios de comunicación, de las cuales, más de ocho horas son dedicadas al Internet, y una de las principales actividades a las que recurren con el $89 \%$, son en las redes sociales. Derivado del uso excesivo de la tecnología, es que se provoca un comportamiento adictivo a tal grado que se ha perdido el desarrollo de otras actividades, como, por ejemplo, hacer actividades al aire libre, pasear, conversar, salir, etc. Algunas manifestaciones adictivas podrían ser: La obsesión por perderse algo de lo que está sucediendo en la red, la adicción al Whatsapp, ansiedad al teléfono celular, la utilización enfermiza del Smartphone, etc. Otros problemas negativos a considerar serían: El estrés tecnológico, sobrecarga de información, multitarea, adicciones y uso indebido de la tecnología. Por otro lado se debe considerar que la Asociación Americana de Psiquiatría no considera como adicción al uso excesivo de la tecnología ya que por ejemplo, no se debe confundir la dependencia del celular con sentirse atraído por las nuevas tecnologías, y el disfrutar de las redes sociales, con ser adicto al internet. Una de las conductas adictivas más estudiadas es la que se relaciona con el Internet, y no hay un diagnóstico fiable o válido que lo considere como un trastorno mental (10).

No obstante, es muy importante que se contemple hacer estudios que definan o evalúen claramente la dependencia o adicción a las nuevas tecnologías, así como estudiar las características psicosociales que pueden actuar como factores de riesgo para la adicción de las nuevas tecnologías. Asimismo, en el ámbito empresarial, el foco de atención debe ser, cómo las organizaciones a través de su cultura organizacional, motivan o inhiben el uso de las tecnologías, y cómo se deben de plantear las nuevas modalidades de trabajo para tener mejores condiciones laborales que no afecten nuestro estado físico y mental (11).

\section{Conclusión}

América latina cuenta con altas tasas de desconexión, al tiempo que no ha sido explotada la potencialidad que Internet y las nuevas tecnologías ofrecen por parte de las naciones, regiones o localidades; por tal motivo se considera que es necesario diseñar ejes estratégicos de desarrollo que incluya una agenda digital con un enfoque global, y que comprometan la acción conjunta de empresas, gobiernos, instituciones educativas, organizaciones civiles y sociedad misma, para que los beneficios de la tecnología permee hacia toda la sociedad, sin anteponer intereses personales o de grupos de poder, y en el que se vea reflejado un verdadero avance en materia de Economía Digital en sus tres componentes: infraestructura de redes de banda ancha, la industria de aplicaciones TIC y los usuarios finales. En México, (12) sugieren que para hacer usufructo de los beneficios de la digitalización es indispensable contar otros factores que van más allá, escogieron tres ejemplos relevantes, un conjunto de agencias reguladoras (IFT, COFECE, PROFECO, CNBV, Banxico, CONDUSEF) independientes coordinadas entre sí, que necesitarán operar a lo largo de la toda la economía para garantizar que la población completa se beneficie de ella, promover la confianza en los sistemas digitales y las transacciones que se realizan a través de ellos y promover la "economía de las aplicaciones" para permitir el desarrollo de herramientas digitales que satisfagan las necesidades locales.

\section{Referencias}

[1] Raygoza, R., Ixmatlahua, S., y Romero, O. (2016). MIMSG: Un Modelo para la Integración de MiPyMES, Sociedad y Gobierno de la zona Metropolitana de Orizaba en el uso de las TIC. RISTI - Revista Ibérica de Sistemas e Tecnologias de Informação, (17), 57-65. Recuperado de https://dx.doi.org/10.17013/risti.17.57-65

[2] Tello, E. (2007). Las tecnologías de la información y comunicaciones (TIC) y la brecha digital: su impacto en la sociedad de México. International Journal of Educational Technology in Higher Education (ETHE), 4(2).

[3] Quiroga, D., Torrent, J., y Murcia, C. (2017). Las tecnologías de la información en América Latina, su incidencia en la productividad: Un análisis comparado con países desarrollados. DYNA, 84(200), 281-290. doi: https://doi.org/10.15446/dyna.v84n200.60632

[4] de León, V., Cerón, M., León, F., y Rodríguez, S. (2016). Impacto de la implementación de la factura electrónica en las MiPyMes del sector comercio y servicios en México. Revista Global de Negocios, 4(7), 8594.

[5] Cedrola, G. (2017). El trabajo en la era digital: Reflexiones sobre el impacto de la digitalización en el trabajo, la regulación laboral y las relaciones laborales. Revista de Derecho de la Universidad de Montevideo, (31).

[6] Ávila, D. (2014). El uso de las TICs en el entorno de la nueva gestión pública mexicana. Andamios, 11(24), 263-288. Recuperado de: 
http://www.scielo.org.mx/scielo.php?script=sci_arttext\&pid=S1870$00632014000100014 \& \operatorname{lng}=\mathrm{es} \& \ln \mathrm{l}=\mathrm{es}$

[7] Leonardi, P., y Neeley, T. (2017). What managers need to know about social tools: avoid the common pitfalls so that your organization can collaborate, learn, and innovate. Harvard Business Review, 95(6), 118126. Recuperado de https://hbr.org/2017/11/what-managers-need-toknow-about-social-tools.

[8] Alva, A. R. (2015). Los nuevos rostros de la desigualdad en el siglo XXI: la brecha digital. Revista mexicana de ciencias políticas y sociales, 60(223), 265-285.

[9] Berrio, C.; Marín, P.; Ferreira, E.; Chagas, E. (2017). Desafíos de la Inclusión Digital: antecedentes, problemáticas y medición de la Brecha Digital de Género. In: Psicología, Conocimiento y Sociedad, Vol 7, Iss 2, Pp 162-198 (2017); (2), 162. doi:10.26864/PCS.v7.n2.8

[10] Natalie, G. (2017). Technology Addiction: How Social Network Sites Impact our Lives. Informing Science The International Journal Of An Emerging Transdiscipline, Vol 20, Pp 179-194 (2017), 179. Recuperado de: $\quad$ http://www.inform.nu/Articles/Vol20/ISJv20p179194Gerhart3423.pdf

[11] Cave, M. \& Flores-Roux, E. (2017). Los beneficios potenciales de la economía digital en México. Consejo Ejecutivo de Empresas Globales, Recuperado de http://ceeg.mx/new/es/2016/01/30/los-beneficiospotenciales-de-la-economia-digital-en-mexico-2/\#.

[12] Soto, A., de Miguel, N., \& Pérez Díaz, V. (2018). Abordaje de adicciones a nuevas tecnologías: una propuesta de prevención en contexto escolar y tratamiento de rehabilitación. Papeles Del Psicólogo, 39(2), 120-126. doi:10.23923/pap.psicol2018.2867 Recuperado de: http://ezproxy.upaep.mx:2382/eds/pdfviewer/pdfviewer?vid=2\&sid=1f 344d3e-9347-4a73-9bef-50a236453962\%40sessionmgr4007 